\title{
Was the number of submissions to scholarly journals in Korea affected by the COVID-19 pandemic?
}

\section{Sun Huh}

Department of Parasitology and Institute of Medical Education, College of Medicine, Hallym University, Chuncheon, Korea

\begin{abstract}
This study investigated whether there was an increase in submissions to scholarly journals in Korea according to journals' field and indexation status in Scopus or Science Citation Index Expanded (SCIE) in 2020, the year when the coronavirus disease 2019 (COVID-19) pandemic first spread throughout the world. The analysis included 60 journals with esubmission systems operated by M2PI. Yearly and monthly submissions were counted from 2016 to 2020. The yearly proportional change was also calculated. In 2020, submissions soared for medical journals indexed in Scopus/SCIE (49.5\%), corresponding to an increase of $36.9 \%$ relative to the expected number of submissions. There was also a surge of submissions to these journals from March to July 2020. However, non-medical journals and medical journals not indexed in Scopus/SCIE did not show an increase in submissions. The number of submissions to scholarly journals in Korea was affected by the COVID-19 pandemic in a specific subset of journals. The background of the spike in submissions is required to be re-investigated. Editors' burden also should be mitigated through editorial board members' help and publishers' support.
\end{abstract}

Keywords

Bibliometrics; COVID-19; Republic of Korea; Scholarly journal; Submission

\section{Introduction}

Received: February 8, 2021 Accepted: February 11, 2021

Correspondence to Sun Huh shuh@hallym.ac.kr

ORCID

Sun Huh

https://orcid.org/0000-0002-8559-8640
Background/rationale: As the editor-in-chief of the Journal of Educational Evaluation for Health Professions, I found that the number of submissions soared from 147 in 2019 to 286 in 2020, reflecting a $94.6 \%$ increase $[1,2]$. Therefore, the burden of the editorial office also increased. I have also heard from some colleague editors that submissions soared in 2020. Altogether, this has been an unexpected change in the environment for editors' voluntary work.

There have also been reports that the number of submissions increased in 2020 for other journals, which some editors interpreted as possibly resulting from the coronavirus disease 
2019 (COVID-19) pandemic. In eight neurosurgical, stroke neurology, and neurointerventional journals, submissions of original articles soared by $42.3 \%$ in 2020 compared to the previous year [3]. In six journals of the British Ecological Society, the number of submissions between March 15, 2019 and October 1, 2019 was compared to the number of submissions during this same period in 2020, and an increase of $15.6 \%$ was found [4]. Compared to the period from February to June 2019, during the corresponding period in 2020 (i.e., from February to June), a 55\% increase in submissions was found for the Journal of Neurosurgery, an increase of $77 \%$ for the Journal of Neurosurgery: Spine, and an increase of 78\% for the Journal of Neurosurgery: Pediatrics [5]. In Annals of Emergency Medicine, the median number of unique articles submitted each month soared by $29.8 \%$ in March 2020, $113.3 \%$ in April 2020, and 133.0\% in May 2020 compared to a baseline period extending from January 2017 to February 2020 [6]. According to a survey of editors, between March 25 and May 1,2020 , submissions increased to 11 journals, whereas no change or a decrease in submissions was reported for four journals [7]. In a survey of Asian editors, 28.9\% of 152 editors reported an increased number of submissions to their journals since the COVID-19 pandemic [8].

What does the soaring number of submissions to scholarly journals in Korea mean? Non-profit societies or organizations publish all but a few scholarly journals in Korea, so the editor's job is a voluntary one without payment. If the number of submissions grows rapidly, editors are required to spend more time reviewing and editing manuscripts. Since editors are usually top-ranking researchers in their fields, and most of them work in universities or hospitals, the soaring number of submissions may induce burnout. Appropriate steps should be taken to help editors. Thus, in light of previous findings and anecdotal reports, it is necessary to confirm whether this phenomenon has also taken place in Korea.

Objectives: This study aimed to determine whether the number of submissions to scholarly journals in Korea was affected by the COVID-19 pandemic. Specifically, the following parameters were analyzed for the journals included in this study: the number of yearly submissions from 2016 to 2020 , the number of monthly submissions from 2016 to 2020, and differences in the number of submissions according to journals' scope and database indexation status.

The following hypotheses were set: first, there was an increase in submissions in 2020 from previous years; and second, the increased number of submissions was confined to medical journals indexed in international databases.

\section{Methods}

Ethics statement: Data were analyzed from the e-submission systems used by journals, the editors of which agreed to provide information for this study. No research data from each individual journal was used. Institutional review board approval and obtainment of informed consent were not required because this study did not have human subjects.

Study design: This was an observational study of the submission count in the e-submission systems operated by a single publishing company. Data were analyzed according to the year, category of journals, and whether journals were indexed in Scopus or Science Citation Index Expanded (SCIE).

Participating journals: Among the 105 journals included in the M2PI's e-submission system, a list was provided of 77 journals that received at least one submission in 2016. Three of these 77 journals were excluded due to a lack of editor information on the journal website. Since the author is an editor of one of those journals, an email was sent to the editors of the 73 other journals to request permission to use the information on their e-submission system. One editor refused to grant permission, and there was no response from 13 editors. Therefore, 60 journals were included in the analysis (81.1\%). Data from these 60 journals were provided by the M2PI.

Data sources/measurement: The number of manuscripts submitted by month and year in the 60 journals was counted. The journals were divided into medical and non-medical fields and then classified according to whether they were listed in Scopus or SCIE. First, the average number of submissions per journal was analyzed in the resulting four groups by year, and the proportional change in the number of submissions by year was calculated. Changes in the number of monthly submissions by group were quantified. Finally, monthly changes in the number of submissions were determined by year in the Scopus/SCIE-indexed journals.

Variables: The variables analyzed in this study were the number of submissions and the journal's category, database indexation status, and year.

Bias: There was no selection bias because this was an observational study of all target journals in a single company's e-submission system.

Study size: Because this was not an experimental study, the number of target journals could not be estimated.

Statistical methods: Comparisons were made by observing the trends of changes. No statistical analysis was done for the comparisons. To estimate the number of submissions in 2020, regression analysis of data from 2016 to 2019 was done with DBSAT ver. 5.0 (DBSTAT Co., Chuncheon, Korea). 


\section{Results}

The number of journals according to field and indexation status in Scopus or SCIE: The list of journals included in this study is available in Suppl. 1. Out of 60 journals, the number of medical journals indexed in Scopus or SCIE was 33 (55.0\%), while the number of medical journals indexed in neither Scopus nor SCIE was 18 (30.0\%). The number of non-medical journals indexed in either Scopus or SCIE was six (10.0\%), while the number of non-medical journals indexed in neither Scopus nor SCIE was three (5.0\%).

Yearly changes in submissions: The yearly changes in submissions are presented in Fig. 1 (Suppl. 2). The rates of change from the previous year are shown in Fig. 2 (Suppl. 2). There was an increase of $49.5 \%$ in the medical journals indexed in Scopus/SCIE. The average expected number of submissions in medical journals indexed in Scopus/SCIE for 2020 estimated from the trends in the previous 4 years was 192. Instead,

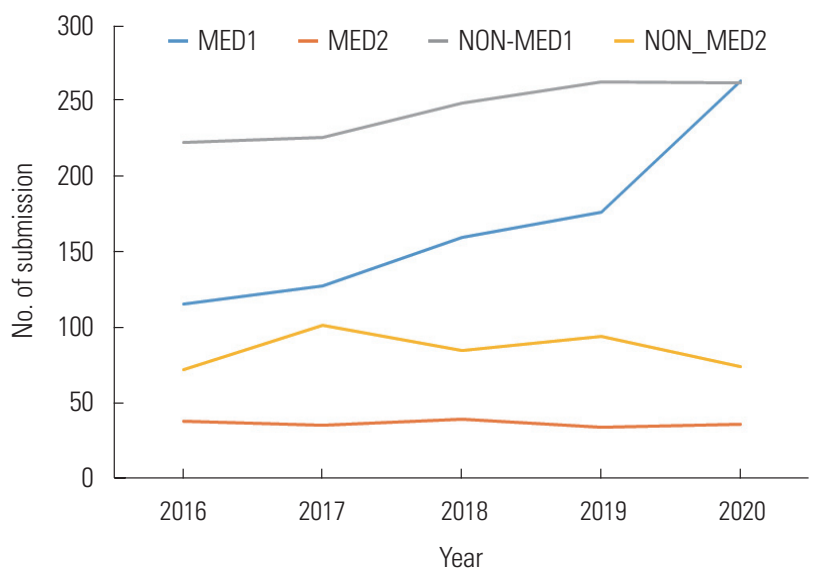

Fig. 1. Average number of submissions to journals in Korea according to research field and database indexation status by year. MED1, medical journals indexed in Scopus or Science Citation Index Expanded (SCIE) (33 journals); MED2, medical journals indexed in neither Scopus nor SCIE (18 journals); NON-MED1, non-medical journals indexed in Scopus or SCIE (six journals); NON-MED2, non-medical journals indexed in neither Scopus nor SCIE (three journals). the real number was 262.8 , reflecting a $36.9 \%$ increase. In contrast, a decrease was observed for the non-medical journals indexed in Scopus/SCIE when the same estimation process was applied (Table 1).

The regression functions of the four groups from 2016 to 2019 were as follows: Medical journals indexed in Scopus/ SCIE: SUBMISSIONS $(\mathrm{Y})=19.700 \times \mathrm{YEAR}(\mathrm{X})+93.500\left(\mathrm{R}^{2}=\right.$ $0.958, \mathrm{P}=0.0210$ )

Medical journals not indexed in Scopus/SCIE: SUBMISSIONS $(\mathrm{Y})=-0.230 \times \mathrm{YEAR}(\mathrm{X})+37.700\left(\mathrm{R}^{2}=0.030, \mathrm{P}=0.8273\right)$

Non-medical journals indexed in Scopus/SCIE: SUBMISSI $\operatorname{ONS}(\mathrm{Y})=14.350 \times \mathrm{YEAR}(\mathrm{X})+203.550\left(\mathrm{R}^{2}=0.944, \mathrm{P}=0.0287\right)$ Non-medical journals not indexed in Scopus/SCIE: SUBMIS $\operatorname{SIONS}(\mathrm{Y})=4.930 \times \mathrm{YEAR}(\mathrm{X})+75.500\left(\mathrm{R}^{2}=0.256, \mathrm{P}=0.4940\right)$

The regression functions of the non-indexed groups were not significantly different $(\mathrm{P}>0.05)$. Therefore, it was not possible to estimate the tendency of submissions by year for these groups.

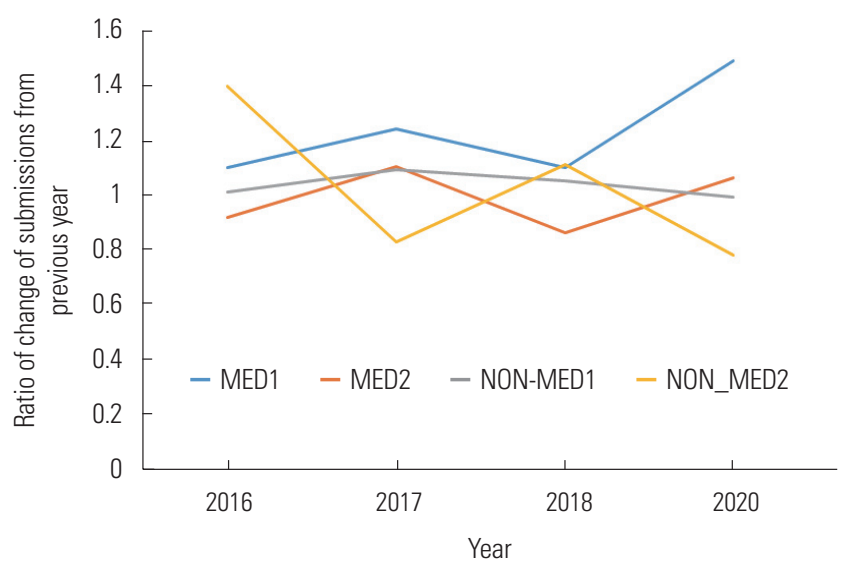

Fig. 2. Annual proportional change in the number of submissions according to research field and database indexation status by year. MED1, medical journals indexed in Scopus or Science Citation Index Expanded (SCIE) (33 journals); MED2, medical journals indexed in neither Scopus nor SCIE (18 journals); NON-MED1, non-medical journals indexed in Scopus or SCIE (six journals); NON-MED2, non-medical journals indexed in neither Scopus nor SCIE (three journals)

Table 1. Changes in the average number of submissions from the expected number extrapolated according to the number of submissions from 2016 to 2019

\begin{tabular}{lrrrrrrr}
\hline & 2016 & 2017 & 2018 & 2019 & 2020 & \multicolumn{2}{c}{$\begin{array}{c}\text { Expected in 2020 } \\
\text { Change relative to the } \\
\text { extrapolated value (\%) }\end{array}$} \\
\hline MED1 & 115.4 & 127.6 & 159.1 & 175.8 & 262.8 & 192.0 & 36.9 \\
MED2 & 38.2 & 35.2 & 38.9 & 33.8 & 36.2 & 36.8 & NA \\
NON-MED1 & 221.8 & 225.7 & 248.0 & 262.2 & 261.5 & 275.3 & -5.1 \\
NON-MED2 & 72.0 & 101.0 & 84.3 & 94.0 & 74.0 & 95.0 & NA
\end{tabular}

MED1, medical journals indexed in Scopus or SCIE (33 journals); MED2, medical journals indexed in neither Scopus nor SCIE (18 journals); NON-MED1, non-medical journals indexed in Scopus or SCIE (6 journals); NON-MED2, non-medical journals indexed in neither Scopus nor SCIE (3 journals). 


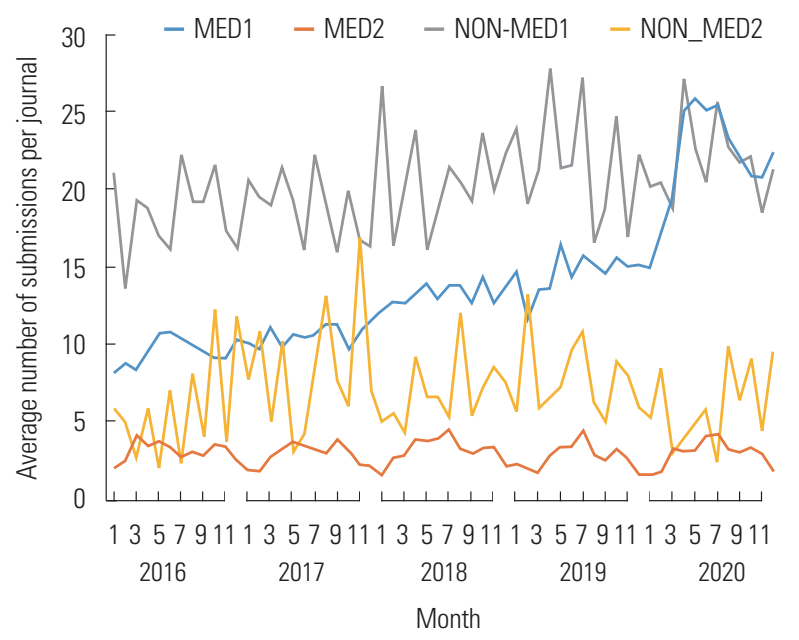

Fig. 3. Average number of submissions to journals in Korea according to research field and database indexation status by month. MED1, medical journals indexed in Scopus or Science Citation Index Expanded (SCIE) (33 journals); MED2, medical journals indexed in neither Scopus nor SCIE (18 journals); NON-MED1, non-medical journals indexed in Scopus or SCIE (6 journals); NON-MED2, non-medical journals indexed in neither Scopus nor SCIE (3 journals).

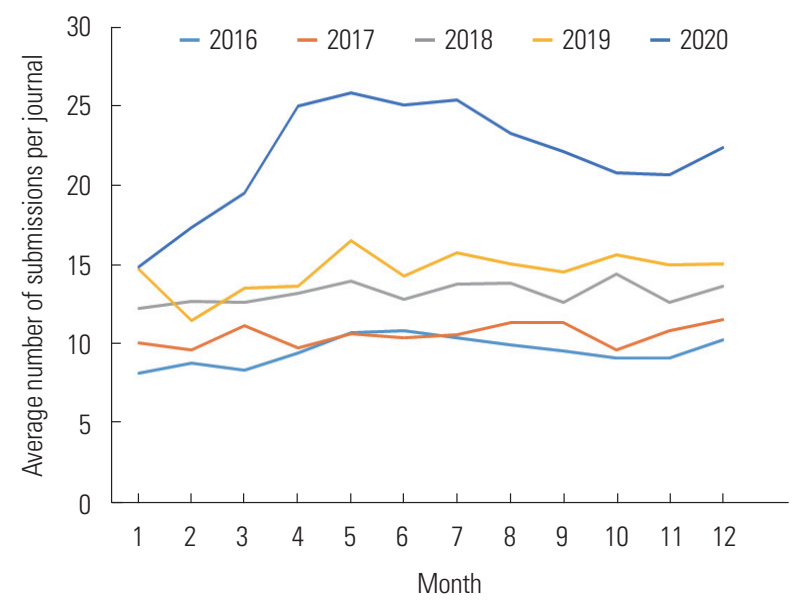

Fig. 4. Average number of submissions to 33 medical journals indexed in Scopus or Science Citation Index Expanded in Korea according to month and year from 2016 to 2020.

The pattern of monthly changes in submissions: The average number of submissions to journals in Korea according to research field and database indexation status by month is shown in Fig. 3 (Suppl. 3). Analyzing the medical journals indexed in Scopus/SCIE, there was no remarkable pattern from 2016 to 2019. However, in 2020, there was a sharp increase from March to April. It persisted to July (Fig. 4, Suppl. 4). There was a fourpeak pattern in non-medical journals indexed in Scopus/SCIE in January, April, July, and October (Fig. 5, Suppl. 5).

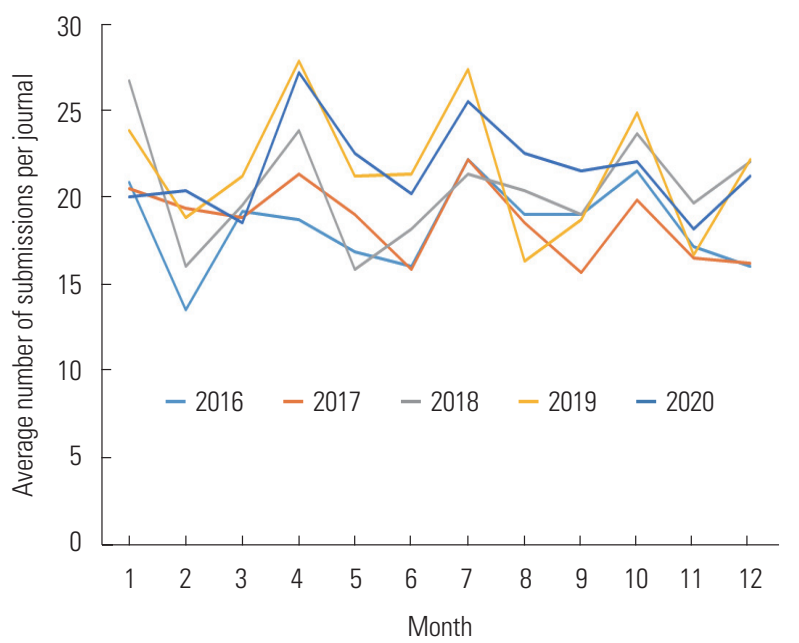

Fig. 5. Average number of submissions to six non-medical journals indexed in Scopus or Science Citation Index Expanded in Korea according to month and year from 2016 to 2020.

\section{Discussion}

Key results: The number of yearly submissions from 2016 to 2020 showed that the spike in submissions was confined to medical journals indexed in Scopus/SCIE (Figs. 1, 2). No meaningful change in the number of submissions was found for non-medical journals or medical journals not indexed in Scopus/SCIE. The pattern of monthly submissions to medical journals indexed in Scopus/SCIE showed an increase from March to July 2020 (Fig. 4).

Interpretation: Why was the increase in submissions limited to medical journals indexed in Scopus/SCIE? This observation is challenging to interpret because no precise information was available on the country of submitters or the content of manuscripts. However, one can speculate about some possible explanations, as follows.

First, since March 2020, due to lockdowns and cancellations of offline scientific meetings, medical researchers may have had more time to write papers. Second, the number of manuscripts on COVID-19 surged in medical journals [9]. Third, submissions increased not only from Korea, but also from outside Korea. The 33 medical journals listed in Scopus/SCIE are all open-access and either do not require article processing charges from authors or have very low article processing charges. Thirty-one of these journals are also indexed in PubMed Central; therefore, they might be suitable journals for many medical researchers throughout the world to consider as possible venues for their research. The same point may hold for Korean researchers. Fourth, researchers in Korea tend to submit their work to Scopus/SCIE journals; therefore, medical journals not indexed in Scopus/SCIE would have 
been unlikely to receive more submissions during the COVID-19 pandemic period. Fifth, in some fields, including the natural sciences, engineering, and agriculture, lockdown measures may have made it difficult to continue experiments or to collaborate with researchers in other countries. The above five speculations can be verified by a more intensive survey of editors or researchers, but for the moment, they must remain hypothetical suggestions.

Some other findings of this study are worth noting. The steady number of submissions to medical journals not indexed in Scopus/SCIE indicates that those journals have been able to continue publishing a consistent number of articles because society members or researchers were loyal to their society journals. The non-medical journals not indexed in Scopus/SCIE received fewer submissions. Therefore, those journals should develop a strategy to recruit more submissions. The monthly submission pattern with peaks in January, April, July, and October in non-medical journals indexed in Scopus/SCIE is difficult to explain. It may reflect random chance. An alternative possibility is that this trend may be due to submission deadlines 2 months before the publication of the issue.

Comparison with previous studies: The increased number of submissions to the 33 medical journals indexed in Scopus/ SCIE (49.5\%) is comparable to the findings of other studies, including a $42.3 \%$ increase in 2020 from the previous year in eight clinical journals [3], and 55\% to $78 \%$ increases in three neurosurgery journals [5]. The monthly patterns of these 33 journals are also comparable to those reported for Annals of Emergency Medicine, which experienced an increase of 29.8\% in March 2020, 113.3\% in April 2020, and 133.0\% in May 2020 from the baseline period [6].

Limitation: Although this study included 60 journals with submission systems operated by one publishing company, it could not represent all scholarly journals in Korea. There were 5,824 Korean scholarly journals as of February 7, 2021, according to the Korea Citation Index database [10]. A random selection of journals would have provided more accurate data. A confounding factor of increased submissions is the inclusion of certain journals in SCIE in 2019, since being indexed in SCIE is usually followed by a surge in the number of submissions to the journal. This phenomenon has frequently been observed for Korean journals [11]. Two journals began to be indexed in SCIE in 2019, but this corresponds to a relatively small proportion (2/33), so it is unlikely that this issue would excessively affect the overall trends. The number of non-medical journals was 15 . Therefore, it would be difficult to conclude that these findings represent the overall status of non-medical journals, although the results are suggestive of some general trends. An editor told me that some manu- scripts were sent directly to the editors (approximately 30 per year) besides those received from the e-submission system. Likewise, this is not a large enough number to affect the overall submission count. Finally, journals were classified only by the author; therefore, differences in opinion regarding the journal classification could be possible.

Generalizability: About 130 medical journals in Korea are indexed in Scopus/SCIE, although the precise number depends on the definition of a medical journal. Thirty-three corresponds to roughly one-fourth of 130 . Therefore, this study may be considered representative of the status of Korean medical journals indexed in Scopus/SCIE.

\section{Conclusion}

This analysis was done to answer the question, "Was the number of submissions to scholarly journals in Korea affected by the COVID-19 pandemic?" The answer is that only medical journals indexed in Scopus/SCIE received more manuscript submissions than expected, with an increase of $36.9 \%$ relative to the expected number of submissions during 2016 to 2019 . There was also a surge in submissions from March to July 2020. These changes might affect editors' daily life by increasing their workload. The number of submissions may also increase in 2021 according to the present trends. Publishers should provide support for editors to overcome this new challenge. Medical academic societies' finances may also be affected by the COVID-19 pandemic, which has made it impossible to hold offline meetings. Editorial board members should share these burdens, and societies should provide financial support for journal editing. To make the review and editorial process more efficient, artificial intelligence tools should also be considered [12].

This study's hypotheses were accepted: there was an increase in submissions in 2020 compared to previous years, and this phenomenon was confined to medical journals indexed in international databases.

\section{Conflict of Interest}

Sun Huh has been the president of the Korean Council of Science Editors since January 2020, but had no role in the decision to publish this case study. M2PI (https://www.m2-pi. $\mathrm{com} /$ ) is a special publishing company member of the Korean Council of Science Editors. It is not for the propagation of the company but for providing submission information in the company's e-submission system. No other potential conflicts of interest relevant to this article were reported. 


\section{Funding}

The author received no financial support for this case study.

\section{Data Availability}

To access each journal's data, please contact the editor of each journal mentioned in Suppl. 1.

\section{Supplementary Material}

Supplementary file is available from the Harvard Dataverse at: https://doi.org/10.7910/DVN/G1W39K

Suppl. 1. List of journals included in this analysis with permission from the corresponding journal editors.

Suppl. 2. Total number, average number, and proportional change of submissions to journals in Korea according to research field and database indexation status by year. MED1, medical journals indexed in Scopus or SCIE (33 journals); MED2, medical journals indexed in neither Scopus nor SCIE (18 journals); NON-MED1, non-medical journals indexed in Scopus or SCIE (six journals); NON-MED2, non-medical journals indexed in neither Scopus nor SCIE (three journals).

Suppl. 3. Average number of submissions to journals in Korea according to research field and database indexation status by month. MED1, medical journals indexed in Scopus or SCIE (33 journals); MED2, medical journals indexed in neither Scopus nor SCIE (18 journals); NON-MED1, non-medical journals indexed in Scopus or SCIE (six journals); NON-MED2, non-medical journals indexed in neither Scopus nor SCIE (three journals).

Suppl. 4. Average number of submissions to 33 medical journals indexed in Scopus or SCIE in Korea according to month and year from 2016 to 2020.

Suppl. 5. Average number of submissions to six non-medical journals indexed in Scopus or SCIE in Korea according to month and year from 2016 to 2020.

\section{References}

1. Huh S. Journal statistics, coping strategy with upcoming scholarly journal publishing environment including Plan$\mathrm{S}$, and appreciation for reviewers and volunteers. J Educ Eval Health Prof 2019;16:41. https://doi.org/10.3352/jeehp. 2019.16.41

2. Huh S. Reflections as 2020 comes to an end: the editing and educational environment during the COVID-19 pandemic, the power of Scopus and Web of Science in scholarly publishing, journal statistics, and appreciation to reviewers and volunteers. J Educ Eval Health Prof 2020;17:44. https://doi. org/10.3352/jeehp.2020.17.44.

3. Lee JE, Mohanty A, Albuquerque FC, et al. Trends in academic productivity in the COVID-19 era: analysis of neurosurgical, stroke neurology, and neurointerventional literature. J Neurointerv Surg 2020;12:1049-52. https://doi.org/10.1136/ neurintsurg-2020-016710.

4. Fox CW, Meyer J. The influence of the global COVID-19 pandemic on manuscript submissions and editor and reviewer performance at six ecology journals. Funct Ecol 2021;35:4-10. https://doi.org/10.1111/1365-2435.13734

5. Kondziolka D, Couldwell WT, Rutka JT. Putting pen to paper during a pandemic: increased manuscript submissions to the JNS Publishing Group. J Neurosurg 2020 Jul 24 [Epub]. https://doi.org/10.3171/2020.7.JNS202691

6. Nigrovic LE, Napper T. Marked escalation in journal submissions during COVID-19 pandemic. Ann Emerg Med 2021;77: 130-1. https://doi.org/10.1016/j.annemergmed.2020.07.003

7. Maghfour J, Olson J, Jacob SE. COVID-19 impacts medical journal submissions. Int J Womens Dermatol 2020;6:255-6. https://doi.org/10.1016/j.ijwd.2020.07.009

8. Chung Y, Kim S, Huh S. Influence of the COVID-19 pandemic on Asian scholarly journal editors' daily life, work, and opinions on future journal development. Sci Ed 2020;7:111-7. https://doi.org/10.6087/kcse.204

9. Else H. How a torrent of COVID science changed research publishing: in seven charts. Nature 2020;588:553. https://doi. org/10.1038/d41586-020-03564-y

10. National Research Foundation of Korea. Number of scholarly journals in Korea [Internet]. Daejeon: National Research Foundation of Korea; 2021 [cited $2021 \mathrm{Feb} 7$ ]. Available from: https://www.kci.go.kr/kciportal/po/statistics/poStatisticsMain.kci

11. Yu JS. Ultrasonography: changes in submission and publication patterns 1 year after being listed in SCIE. Ultrasonography 2021;40:1-2. https://doi.org/10.14366/usg.20184

12. Kim K. Artificial intelligence and publishing. Sci Ed 2019;6:8990. https://doi.org/10.6087/kcse.168 